Supporting Information

\title{
Multifunctional and Continuous Gradients of Biointerfaces Based on Dual Reverse Click Reactions
}

Zhen-Yu Guan, Chih-Yu Wu, Jyun-Ting Wu, Ching-Heng Tai, Jiashing Yu, and Hsien-Yeh Chen*

Department of Chemical Engineering, National Taiwan University, Taipei 10617 (Taiwan)

Corresponding Author: hsychen@,ntu.edu.tw 


\section{Reaction Kinetics of the Thermo-Activated Thiol-Yne Click Reaction}

The reaction rate of the thiol-yne click reaction by propiolate or alkyne was found to follow first-order kinetics ${ }^{[12]}$ and can be expressed as follows:

$\frac{d[C \equiv C]}{d t}=-k[S H]=-A \cdot e^{-E / R T} \cdot[S H]$

where $[C \equiv C] / d t$ is the depletion of the propiolate concentration, which can also be termed the degree of the conversion for the reaction. $[S H]$ is the concentration of the thiol molecules in the system; $k$ is the rate constant and can be rewritten following the Arrhenius equation $A \cdot e^{-E / R T}{ }^{[23]}$ where $A$ is the pre-exponential factor and $E$ is the activation energy. Based on the assumption of a constant concentration of thiol molecules in the system and the first-order reaction kinetics for the thiol-yne reaction, $[C \equiv C] / d t$ depends linearly on only the rate constant and is thus proportional to the value of $e^{-1 / T}$.

\section{IRRAS analysis}

The normalized $-\mathrm{NH}$ band intensity from the IRRAS results is representative of the reaction conversion, and the band intensity was plotted with respect to the temperature gradient used in the study (from 10 to $90{ }^{\circ} \mathrm{C}$ ) and was calculated according to the aforementioned rate equation. The data points were also found to be in good agreement with the trajectory of $e^{-1 / T}$. The normalization was performed using the peak intensity of the $-C H$ band, and the reaction conversion was expressed as $I_{N H} / I_{N H}{ }^{*}$, where $I_{N H}$ is the normalized -NH band intensity of the gradient surface and $I_{N H}{ }^{*}$ is the normalized $-\mathrm{NH}$ band intensity of the homogeneously immobilized Cy5RGDACC, which assumed complete reaction. The activation energy $E_{\text {IRRAS }}=2.86 \times 10^{4} \mathrm{~J} / \mathrm{mol}$ by the IRRAS analysis can thus be calculated from the logarithmic slope, and the pre-exponential factor $A_{I R R A S}=1.26 \times 10^{4}$ can be determined from the intercept.

\section{Fluorescence analysis}

Similarly, the fluorescence intensity from Cy5-RGDACC is also indicative of the reaction conversion, and such intensity was plotted with respect to the temperature gradient and was calculated according to the aforementioned rate equation. The data points were also found to be in good agreement with the trajectory of $e^{-1 / T}$. The activation energy $E_{\text {fluorescence }}=2.80 \times 10^{4} \mathrm{~J} / \mathrm{mol}$ from the fluorescence analysis can thus be calculated from the logarithmic slope, and the pre-exponential factor $A_{\text {fluorescence }}=1.00 \times 10^{4}$ can be determined from the intercept. The results of the activation energy and the pre-exponential factor calculated from the IRRAS and fluorescence analyses presented consistent values, thereby unambiguously verifying the dependence of the reaction conversion (reactivity) on the temperature gradient $e^{-1 / T}$.

Additionally, the immobilization of Alexa Fluor ${ }^{\circledR} 555$ azide molecules via the copper-free alkyne and azide click reaction followed the gradient of the complementary reactivity of unreacted propiolates, and the fluorescence intensity from Alexa Fluor ${ }^{\circledR} 555$ was also plotted with respect to the temperature gradient. The data points were found to fit well with the trajectory of $1-e^{-1 / T}$, thus verifying the hypothesis of complementary reactivity. 


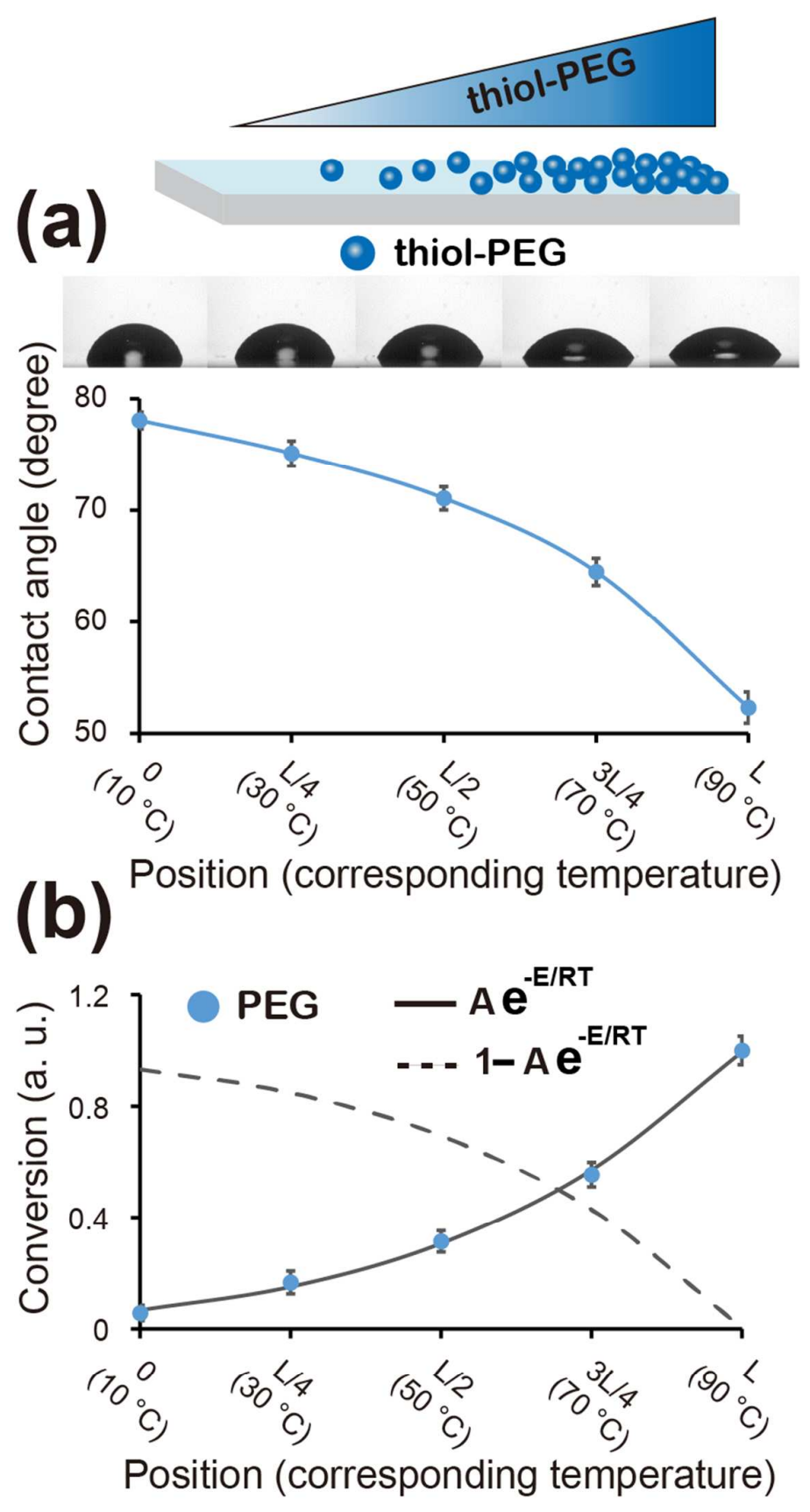

Figure S1. Examination of water contact angle (CA) on the PEG gradient. (a) Images of CA measurement along various positions with respect to $L$; the $C A$ values were plotted against the various positions, showing a decreasing and gradient distribution along L. (b) The conversion of PEG immobilization was calculated based on the hypothesis that increasing PEG amount is complimentarily dependent on the decrease in CA value (increased hydrophilicity). The calculated PEG conversion fit well to the first-order kinetics of the thiol-yne click reaction (described above) and showed consistency with the trajectory of $\mathrm{e}^{-1 / \mathrm{T}}$. 


\section{(a) PEG-only}

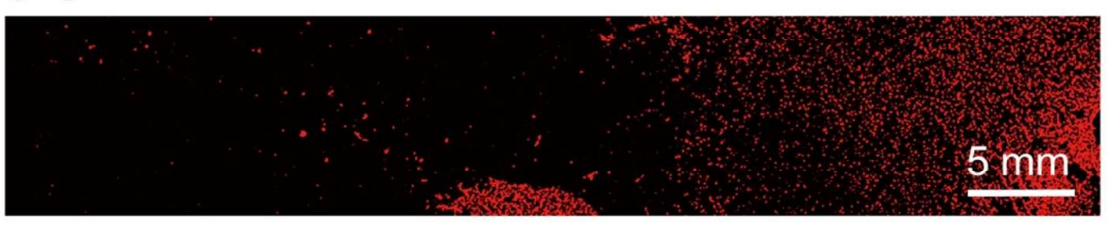

\section{(b) countercurrent gradient with PEG and RGD}

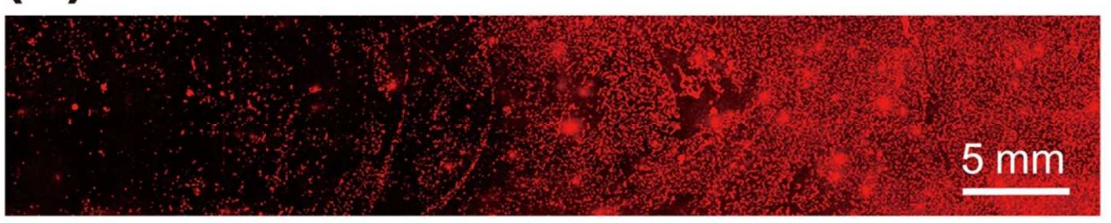

Figure S2. Fluorescence images of 3T3 cell adhesion study on the gradient surface of (a) PEG-only and (b) countercurrent gradients of PEG and RGD. Cells were stained using NucRed ${ }^{\mathbb{B}}$ Live 647 ReadyProbes ${ }^{\mathbb{B}}$ Reagent. The images were used for the statistical analysis, and the results were discussed in the paper. 


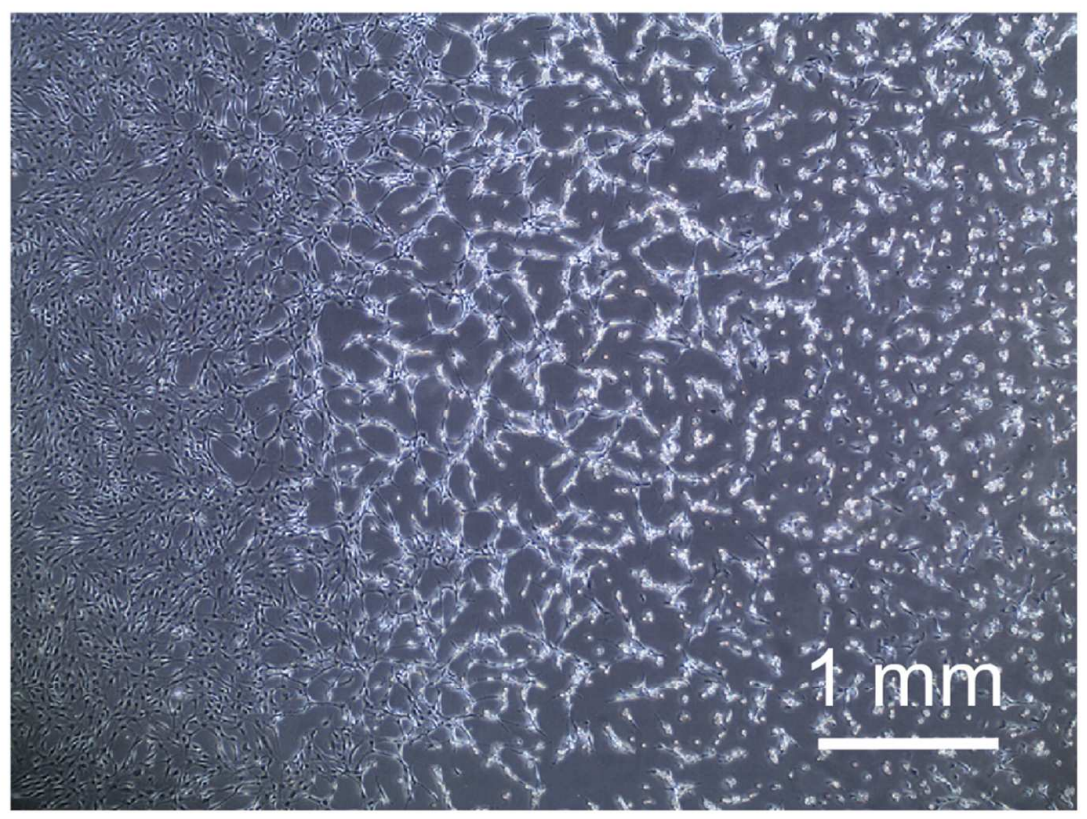

Figure S3. One-shot micrograph of $3 \mathrm{~T} 3$ cell adhesion recorded on a surface containing countercurrent gradients of PEG and RGD. The image contains a field of view of $6 \mathrm{~mm} \times 4.5 \mathrm{~mm}$. 


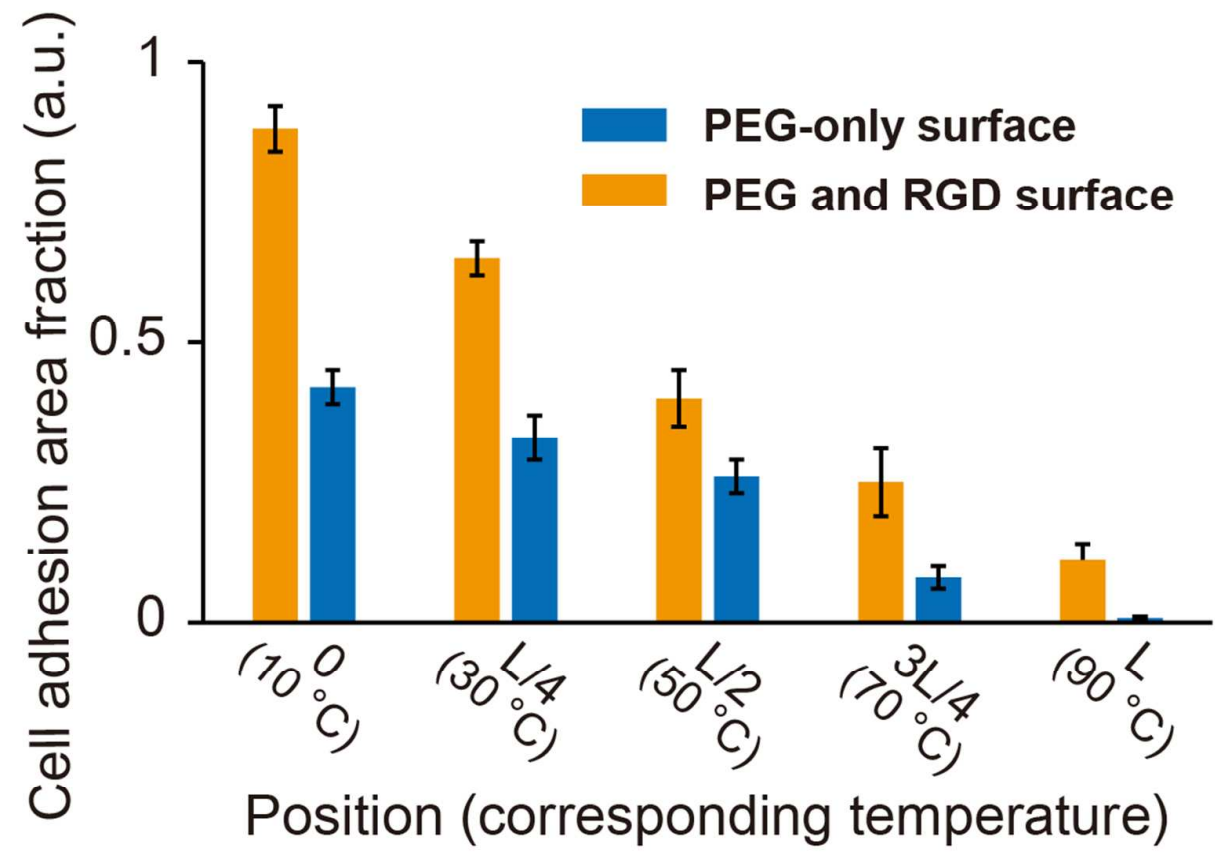

Figure S4. Analysis of 3T3 cell adhesion area fraction on surfaces containing only PEG gradient or the countercurrent gradients of PEG and RGD. A gradient variation of cell adhesion from $\sim 0.01$ to 0.42 was found for the PEG-only surface, and an enhancement of the gradient distribution from 0.11 to 0.88 was discovered for the PEG/RGD surface. Error bar represents the mean values $( \pm$ SD) of three independent experiments. 


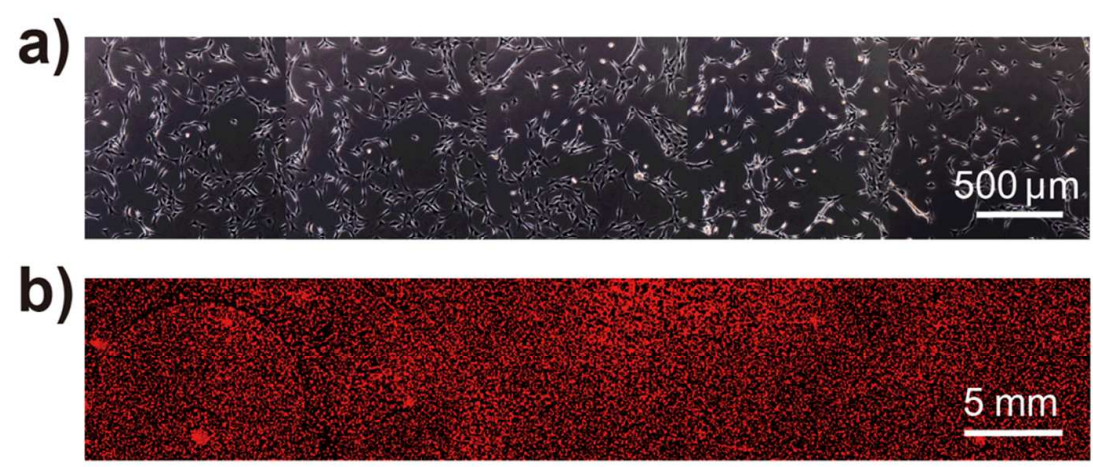

Figure S5. Controlled experiments of 3T3 cell adhesion study on as-deposited coating of poly[(4-methylpropiolate- $p$-xylylene)-co-( $p$-xylylene)]. (a) Image of resulting cell growth pattern captured using an optical microscope. (b) Fluorescence image of the resulting cells stained using NucRed ${ }^{\circledR}$ Live 647 ReadyProbes $^{\circledR}$ Reagent. The results revealed homogeneous attachment and growth of 3T3 cells. 


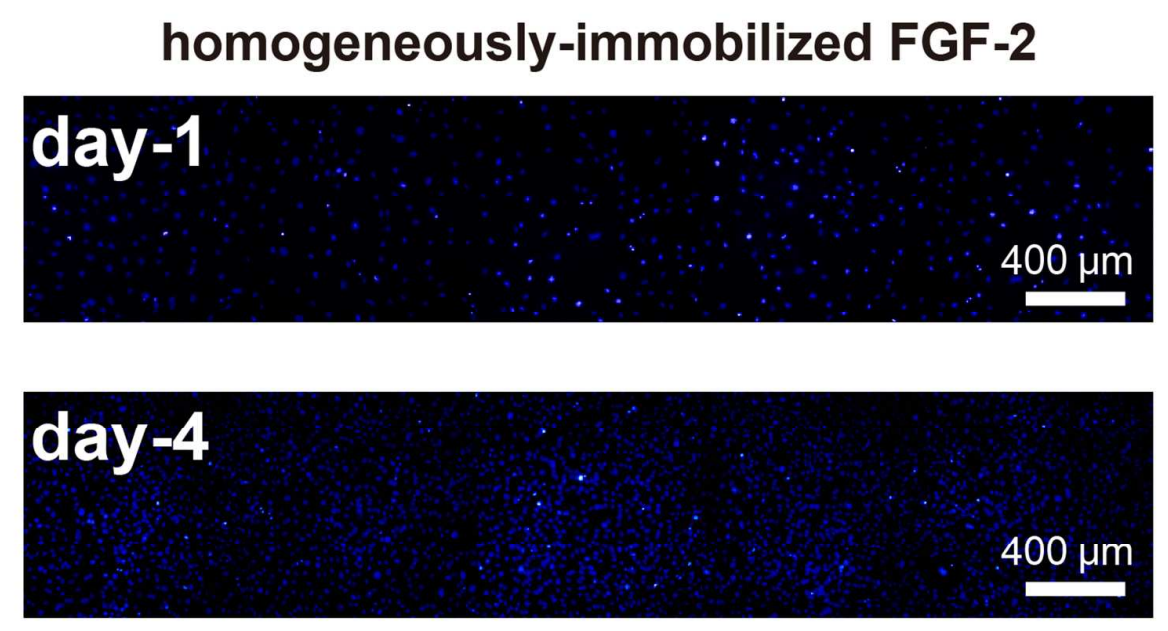

Figure S6. Controlled experiments of ASC proliferation examined on homogeneously immobilized FGF-2 surface at day 1 and day 4 . Homogeneous growth and proliferation patterns were observed throughout the entire sample surface. 


\section{homogeneously-immobilized BMP-2}

\section{a) ALP expression (day-10)}

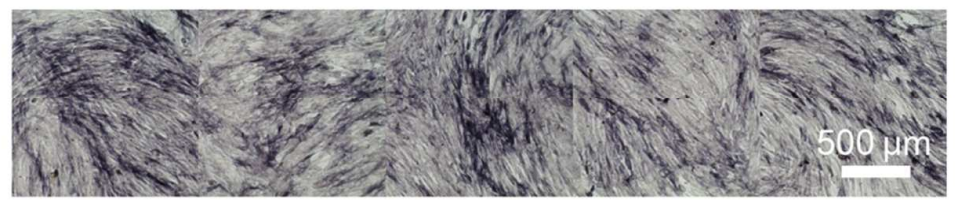

\section{b) calcium deposition (day-21)}

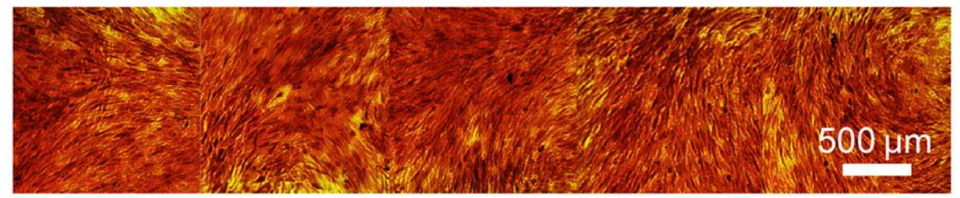

\section{c) osteocalcin expression (day-21)}

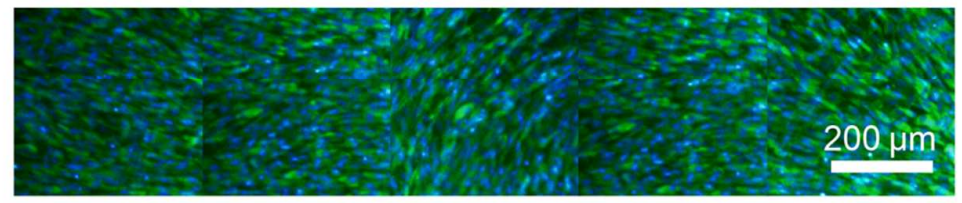

Figure S7. Controlled experiments for examining the osteogenesis activities for ASCs on surfaces with homogeneously immobilized BMP-2. (a) ALP expression was analysed at day 10, (b) calcium deposition using Alizarin red was examined at day 21, and (c) osteocalcin expression detected using immunofluorescence staining of fluorescein-labelled goat anti-rabbit IgG was performed at day 21. Homogeneous distributions of these osteogenesis activities were observed throughout the entire sample surface. 